\title{
Why dexamethasone can reduce mortality rate among patients with viral infections
}

Yuhan Lin ${ }^{1}$, Lipeng Liao ${ }^{1}$, Ying Zhang ${ }^{2}$, Chanyuk Lam ${ }^{1}$, Man Tang ${ }^{1}$, Qiuyun Liu ${ }^{1}$ ${ }^{1}$ School of Life Sciences, Sun Yat-sen University, Guangzhou 510275, China.

${ }^{2}$ Guangzhou Center for Disease Control and Prevention, Guangzhou 510440, China.

$\triangle$ Correspondence: Qiuyun Liu, Ph.D, School of Life Sciences, Sun Yat-sen University, Guangzhou 510275, China. Tel: $\left[\begin{array}{llll}+86 & (020) & 84110296\end{array}\right]$ (lsslqy@mail.sysu.edu.cn). 


\begin{abstract}
As an anti-inflammatory hormone, dexamethasone may minimize proton buildup and down-regulate the production of oxalate. The reduced generation of insoluble and stiff calcium oxalate might account for the substantial drop of mortality rates by dexamethasone amid the current COVID-19 pandemic.
\end{abstract}

Keywords

Dexamethasone; proton buildup; down-regulate; insoluble and stiff calcium oxalate 
Dexamethasone can reduce fever, perhaps via the decrease of proton traffic and proton pool, thus attenuating the production of ATP through the respiratory chain. Oxalate production or traffic may be induced or affected by high proton level respectively, and the insoluble and stiff calcium oxalate can counteract strong acids such as $\mathrm{HCl}$ derived from excess of protons. Calcium oxalate is stressful to cells and is the primary component of renal stones which can advance to kidney failure and shorten lifespan [1]. To make matters worse, cancer and other patients might overproduce calcium oxalate to antagonize strong acids resulted from proton stress [2-4]. Calcium oxalate crystals were found in the thyroids of over $85 \%$ of the patients 70 years of age and above within 5 hours post-death [5]. The proteins of SARS-CoV-2 are high in valine plus glycine [2,6], while glycine can chelate divalent cations [7]. Valine is also likely to bind calcium and other divalent cations [6,8]. The extensive secondary chemical bonding between calcium and valine and glycine residues in proteins enhances the formation of insoluble calcium salts which might be the trigger of lung stickiness and breathing difficulty in COVID-19 patients.

As an anti-inflammatory agent, dexamethasone can minimize the buildup of protons and might down-modulate the generation of oxalate, and the reduced production of calcium oxalate may account for the substantial reduction of mortality rates by dexamethasone during the COVID-19 pandemic [9]. The beneficial or negative effects of dexamethasone should be comprehensively studied and assessed to further our understanding on the mode of action of this hormone.

Conflict of interest The authors declare that they have no conflict of interest.

Acknowledgements This work was supported by grant from Guangzhou Science and Technology Program (201804010328) to Q. Liu; National Science and Technology Major Project of the Ministry of Science and Technology of China (2017ZX10103011) to Y. Zhang. We thank Yan Shi for editing.

Author Contributions QL conceived the study and supervised the work. All authors contributed to the gathering of references, and contributed to the analysis, interpretation and preparation of the manuscript.

\section{References}

1. Hall JA, Yerramilli M, Obare E, Li J, Yerramilli M, Jewell DE. Serum concentrations of symmetric dimethylarginine and creatinine in cats with kidney stones. PLoS One. 2017;12(4):e0174854. https://doi.org/10.1371/journal.pone.0174854.

2. Yan S, Zhang Y, Liu Q. Why is COVID-19 virus so deadly for cancer $\begin{array}{llll}\text { patients?. Eur J } & \text { Cancer }\end{array}$ https://doi.org/10.1097/CEJ.0000000000000605. 
3. Du Y, Fu M, Peng J, An S, Liu Q. How to alleviate cancer-caused secondary heart $\begin{array}{llll}\text { disease. Eur J Prev Cardiol. } & \text { 2018;25(15):1675. }\end{array}$ https://doi.org/10.1177/2047487318782510.

4. An S, Li X, Tang M, Yan S, Yang F, Zhu X, et al. Functional duality of ethanol on cancer. Med Hypotheses. 2019;122:124-5. https://doi.org/10.1016/j.mehy.2018.11.007.

5. Katoh R, Suzuki K, Hemmi A, Kawaoi A. Nature and significance of calcium oxalate crystals in normal human thyroid gland. A clinicopathological and immunohistochemical study. Virchows Arch A Pathol Anat Histopathol. 1993;422(4):301-6. https://doi.org/10.1007/BF01608339.

6. Wan Y, Yan S, Zhang Y, An S, Yang K, Xu H, et al. The pneumonia outbreak: high isoleucine and high valine plus glycine contents are features of the proteins of COVID-19 virus. Preprints. 2020;2020020289. doi: 10.20944/preprints202002.0289.v5.

7. Wang Y, Tang JW, Ma WQ, Feng J, Feng J. Dietary zinc glycine chelate on growth performance, tissue mineral concentrations, and serum enzyme activity in weanling piglets. Biol Trace Elem Res. 2010;133(3):325-34. https://doi.org/10.1007/s12011-009-8437-3.

8. Strasdeit H, Büsching I, Behrends S, Saak W, Barklage W. Syntheses and properties of zinc and calcium complexes of valinate and isovalinate: metal alphaamino acidates as possible constituents of the early Earth's chemical inventory. Chemistry. 2001;7(5):1133-42. https://doi.org/10.1002/15213765(20010302)7:5<1133::aid-chem1133>3.0.co;2-t.

9. Horby P, Lim WS, Emberson JR, Mafham M, Bell JL, Linsell L, et al. Dexamethasone in Hospitalized Patients with Covid-19 - Preliminary Report. N Engl J Med. 2021; 384:693-704. https://doi.org/10.1056/NEJMoa2021436. 